\title{
Sleep Apnea Monitoring Using Mobile Phones
}

\author{
Shamma Alqassim, Madhumeta Ganesh, Shaheen Khoja, Meher Zaidi, Fadi Aloul, Assim Sagahyroon \\ Department of Computer Science \& Engineering, American University of Sharjah, UAE
}

\begin{abstract}
Obstructive Sleep Apnea (OSA) is a sleeping disorder characterized by the repetitive reduction of airflow during sleep. In this paper, we discuss the design and implementation of a user-friendly mobile application developed on multiple platforms (Windows and Android) to monitor and detect symptoms of sleep apnea using the smart phone's built-in sensors. The purpose of the application, Sleep Apnea Monitor (SAM), is to allow users to get a sense of whether or not they are likely to have sleep apnea, before continuing with more expensive and advanced sleep tests. In addition, SAM provides doctors and sleep specialists with remote access to patients' records and allows them to confirm their initial diagnosis. The parameters measured by this application are breathing patterns and movement patterns, which are recorded respectively using the built-in microphone and accelerometer. The recorded data is sent to a server for analysis in order to diagnose patients and maintain geographical studies of areas with sleep apnea patterns. The application is successfully tested among a number of users in the UAE. The system diagnoses and reports the level of the user's sleep apnea. In addition, doctors can remotely monitor users through the website, which is interfaced with Google Maps to keep track of user locations, and keep track of their analysed records.
\end{abstract}

\section{INTRODUCTION}

As reported in recent studies [1], the utilization of hand held devices such as smart phones in health-related applications will continue to increase. The rich features that today's smart phones are equipped with provide industry and researchers with a valuable opportunity to continue improving human life by developing applications that address a wide spectrum of issues. Applications that address sleeping disorders have been reported [2] and such applications focus on a wide range of disorders, including sleep apnea, mostly by utilizing the built-in features and sensors of the phone. However, although helpful, many of these systems do not provide users with a comprehensive overview of the disorder, as they tackle a single symptom rather than incorporating multiple indicators for a more conclusive diagnosis.

Sleep apnea is a sleep disorder characterized by the repetitive reduction of airflow during sleep, which in turn causes pauses and reduced breathing. These recurring arousals from sleep due to a blockage of airway cause fragmented sleeping patterns and lead to the activation of the body's sympathetic nervous system. An apnea is defined as the duration of time when there is complete blockage of airflow for 10 seconds or more, and is measured in apnea-hypopnoea index (AHI) [3].

Some of the symptoms that sleep apnea patients commonly display include snoring, pauses in breathing during sleep, choking or gasping for air following breathing disturbances, daytime sleepiness while carrying out routine tasks, headaches, dryness of throat in the morning, lack of concentration ability, urination at night, depression and irritability, and obesity [4].

According to the World Health Organization approximately 100 million people worldwide have obstructive sleep apnea (OSA). In the United States, OSA is estimated to affect 1 in 4 men and 1 in 9 women; it also affects 23 million working adults [5]. Approximately $4 \%$ of men and $2 \%$ of women over the age of 35 years have symptomatic moderate or severe OSA, affecting approximately 12 million people in the United States. It is estimated that less than $25 \%$ of OSA sufferers have been diagnosed [5].
The diagnosis of OSA can be done through a number of different tests and techniques. However, the symptoms of the OSA disorder are not very specific and may sometimes be caused by factors other than sleep apnea such as alcohol intake, lack of sleep and stress. Therefore, sleep specialists need to perform appropriate tests to rule out and distinguish these symptoms. If these results suggest sleep apnea, then further diagnostic tests need to be performed, which include medical, physical and sleep history exams [6].

Once the probability of OSA is determined to be high from the conducted tests, then a polysomnography is usually conducted. Polysomnography is the most common sleep study that is performed overnight and measures the patient's brain activity along with a number of other measurements. It monitors electronically the sleep stages, measures eye movements, brain waves, respiratory airflow and effort, changes in breathing, blood oxygen levels and heart rate. The doctor will then measure the number of apneas that occurred and that lasted over 10 seconds. Significant apnea is diagnosed if over 5 apnea episodes occur during an hour [7].

Although polysomnography is the most effective method of diagnosis, it is highly expensive and requires a great deal of dedicated resources. As a result, a number of less expensive and simpler techniques are being developed to diagnose OSA including split night polysomnography and home sleep studies. These suggested methods may not be as accurate but can be used as a starting point or alternative to overnight polysomnography [8].

Unlike existing applications which consider only partial signs of sleep apnea, the proposed application's approach is to use the built-in sensors to collect data representing critical parameters for a proper initial diagnosis. By using the built-in sensors, this application seeks to collect a representative set of data that, upon analysis, will assist in identifying, with a high degree of certainty, whether or not sleep apnea symptoms exist.

The rest of the paper is organized as follows. Section II specifies the literature review and related work conducted during the project. Section III describes the system hardware and the software architecture. The system requirements are discussed in Section IV. The testing and implementation results are reported in Section V. Finally, the conclusion is presented in Section VI.

\section{RELATED WORK}

Several studies have been presented and examined in the field of obstructive sleep apnea. MedAssist is a sleep apnea monitoring system, consisting of an external electrocardiography (ECG) sensor, a microcontroller, an Android computing platform and a server that identifies OSA episodes. The system was tested using 35 subjects, whose recordings were divided into 1-min segments. Each minute was classified as either "apnea minute" or a "non apnea minute" on the basis of respiration and oxygen level scored by experts [9]. These recordings were classified into 3 groups: Group A with high degree of sleep apnea (100 minutes or more), Group B with a moderate degree of sleep apnea ( 5 to 99 minutes) and Group $\mathrm{C}$ that has no sleep apnea (below 5 minutes) [9]. While MedAssisst examines the electrical activity of the heart during sleep time and categorizes the apnea and non apnea minutes, the proposed solution SAM diagnoses sleep apnea using breathing patterns (that may consist of snoring which is an indicator of sleep apnea) and movement recordings instead of heart rate. Two other studies presented in [10] and [11] propose methodologies and tools to differentiate simple snoring from the snoring of sleep apnea patients. 


\section{SYSTEM ARCHITECTURE}

The system hardware and software architecture respectively describe the structure and behaviour of the Sleep Apnea Monitor application (SAM).

\section{A. System Overview}

The proposed solution consists of the development of a mobile application that uses voice recording software and the mobile's builtin accelerometer to record the users' breathing patterns and movements during their sleep. In addition, the application allows for determining the location of the patients using the built-in GSM cell tower triangulations. As shown in Figure 1, the recorded data is securely sent to a remote server using Wi-Fi or $3 \mathrm{G}$ connection. On the server side, statistical analysis of data is performed to derive indicators that would assist in diagnosing patients' condition. After the processing of data is completed and the analysis is concluded, the results are securely sent back to the user's phone and stored on the server's database as well. This database is accessible via a web server by the doctors and medical staff with privileged access to users' records. After the data is examined by the physician and the diagnosis is complete, a text message may be sent to the user with the outcome.

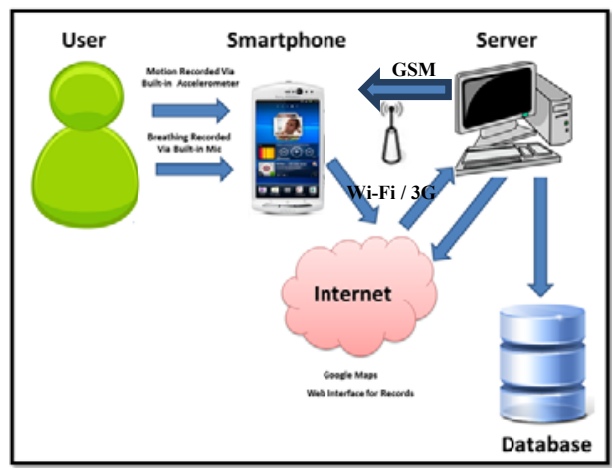

Figure1. Sleep Apnea Monitoring System Overview.

\section{B. System Hardware}

\section{Smart Phone}

The smart phones used in this work are the Android-based Sony Xperia neo V (OS 2.3.4) and Windows-based HTC 7 T8698 Mozart (OS 7.0), both of which have a processing speed of $1 \mathrm{GHz}$ and are equipped with a built-in microphone, accelerometer, GPS receiver and Wi-Fi capabilities. The recordings of the Android phone are stored in the SD card, while the recordings of the Windows phone are stored in the isolated storage of the phone since all I/O operations on Windows are restricted to this storage.

\section{Server/GSM Modem}

The server used has a processing speed of $2.66 \mathrm{GHz}$ and $2 \mathrm{~GB}$ of RAM. The GSM Modem used is the Siemens MC35i module. SMS alerts and diagnosis messages are sent by physicians to patients using this modem.

\section{Software Architecture}

Different software tools are used to develop SAM, as shown in Figure 2.

\section{Programming the Smart Phones}

The android-based phone application is developed using the Java programming language within Eclipse IDE, with support from the Android Development Tools. On the other hand, the Windows-based phone is developed using Visual Studio 2010, with the Windows Phone SDK tools.

Both applications have the following features:
- Allow users to register if they are using the application for the first time.

- Allow users to enter their personal unique username and password to access their accounts and begin with the data logging and recording.

- Permit sound and accelerometer recording via the smartphones' inbuilt features.

- Allow saving of the audio recording as .amr (Android)/.wav (Windows) format and accelerometer readings as a .csv file.

- Allows saving of the geographical location of the mobile phone location, retrieved from the Google Maps API (for Android) and Bing Maps (for Windows), which is based on GSM cell tower triangulation.

- Allow the secure sending of the recorded data via Wi-Fi to the server, using HTTPS requests (POST and GET).

- Receive the preliminary processed results as well as a message containing the Doctor's comments via SMS.

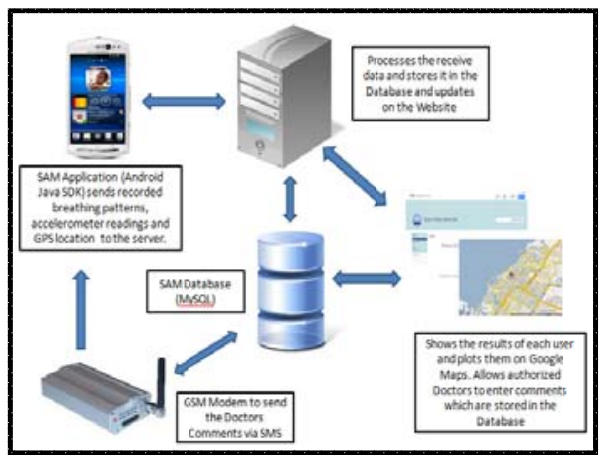

Figure 2. Software Architecture.

2. Database

A database management system (MySQL) is used to store the users' and doctors' information. The database is also used to store current and previous patients' history. The system database consists of the following major tables:

- User_Details: Records the user information when they first register.

- Doctor_Details: Stores the authorized Doctor's details.

- Apnea_Details: Stores the results of the processing for each user. New entries are inserted every time the data sent by the user is processed on the server.

\section{Website}

The SAM website has been developed using Google Sites, PHP and HTML. Using an easy-to-use web portal with a friendly interface, doctors are able to retrieve posted results, analyse them and post back their comments and conclusions. Additionally, the website implements Google Maps functionality to allow authorized doctors to view the locations of the users.

\section{Communicating with the Server}

The analysis of the snoring pattern and body motion on the server is carried out on the server. The PHP programming allows the phone to connect to the server so that the data can be sent over Wi-Fi/3G for processing. After processing, the results are sent back to the phone, while simultaneously being stored in the database as well as displayed on the website. The server we are using is the WAMPSERVER 2.0c which is an open source server that allows PHP programming and easy connection to a MySQL database.

\section{SMS Gateway Application}

The Ozeki Server Manager and Ozeki Server Monitor are used to set up the GSM Modem and provide a simple and easy-to-use GUI interface to compose and send SMS and monitor the status of messages. In our system, the message consists of the doctor's opinion 
on whether the patient is likely to have sleep apnea symptoms or not based on the analysis of the accelerometer and snoring pattern graphs.

\section{Signal Processing Aspects}

MATLAB is used to process the voice and movement recordings that are received by the server from the smartphone. The server receives the sound file in amr format for the Android phone or in .wav for the Windows phone. It also receives a .csv file for the movements. For the android, the web server then calls a PHP page that converts the audio file to .wav format using the ffmpeg converter (the converting does not take place for the Windows phone, as the file received is already a .wav file). Short-time Fourier transform analysis is performed to obtain the frequency spectrum of the signal. The frequencies at the maximum spectral amplitude of the wave file are found and recorded in an array. In [11], the threshold of $800 \mathrm{~Hz}$ is suggested to be a reference. Hence, the number of instances where the frequency has exceeded $800 \mathrm{~Hz}$ is used to calculate the percentage of sleep time the user was snoring. A graph of frequency vs. time is plotted to show the breathing pattern of the user during the sleep study. Figure 3(a) shows the image of the amplitudes of the audio signal in the frequency vs. time domain which was used to extract the frequency vs. time graph in 3(b). Both show a breathing pattern graph where the user snored six times in 12 seconds. The spikes (known as sleep apnea snoring minutes) crossing the $800 \mathrm{~Hz}$ pink threshold represent the snores.
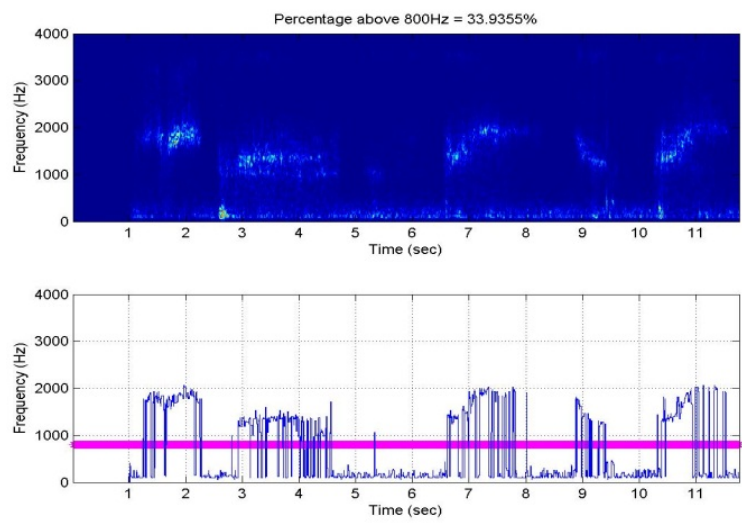

Figure 3.(a) Audio signal's amplitude in the frequency vs. time domain and (b) frequency vs. time domain graph extracted from (a).

Subsequently, the accelerometer data (consisting of $x, y$ and $z$ components of the acceleration of the body movement in units of $G$ where $G$ is a unit of acceleration) is imported into MATLAB. The root-sum-square method (RSS) is then used to calculate overall change in acceleration by using the three columns of data ( $x, y$ and $z$ values) in the .csv file [12]. This method squares each corresponding $x, y$ and $z$ value separately, adds them and finds their square root.

$$
\mathrm{RSS}=\mathrm{SQRT}\left(\mathrm{x}^{2}+\mathrm{y}^{2}+\mathrm{z}^{2}\right)
$$

This method was used because the resultant acceleration vector is composed of $x, y$ and $z$ components and, in order to find its magnitude, each value needs to be squared and the total needs to be square-rooted. The RSS value does not change when the object is stationary and only changes when there is change in one, two or all of the components of acceleration [12]. Hence, when the user's body remains stationary, this means the person is breathing normally and is not experiencing sleep apnea and his movement graph will show a horizontal line at $1 \mathrm{G}$.

If the person moves during the sleep study, this may be because the person is experiencing sleep apnea and is tossing and turning a lot in his/her sleep; all these movements are recorded in the form of spikes in the accelerometer graph. The threshold of $1.03 \mathrm{G}$ (obtained by testing the code with files that consisted of no movement, minimal movement and lots of movement) was set in the code and RSS points that cross this threshold were recorded. Finally, a percentage was calculated based on how many of the total number of points crossed this threshold.

Figure 4 shows an accelerometer graph where the user's hand moved upwards twice in 12 seconds. The spikes crossing the threshold are known as sleep apnea movement minutes.

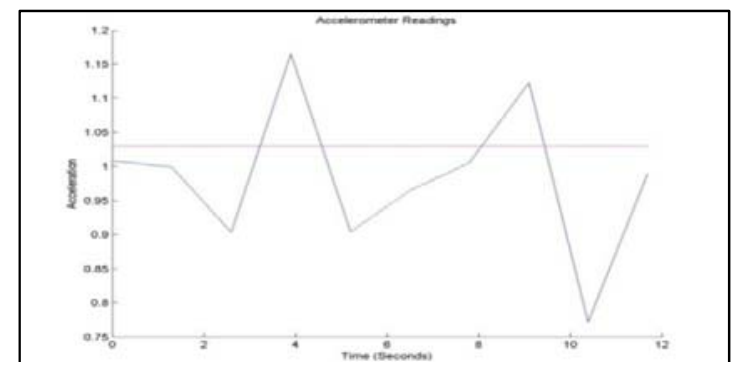

Figure 4. Movement pattern graph.

The final sleep apnea percentage (or sleep apnea score) of the user is then calculated on MATLAB by taking a weighted percentage of the sleep apnea movement minutes and previously calculated sleep apnea snoring minutes. The application will compare the user's sleep apnea score to the physician approved numbers and deduce if the patient is experiencing no sleep apnea, mild sleep apnea or severe sleep apnea.

The output files, which are two graphs and a file with the percentage of sleep apnea, are sent back to the users' mobile application and subsequently stored on the database for future references.

\section{SYSTEM REQUIREMENTS}

A system can be characterized according to its functional and nonfunctional requirements. Functional requirements describe the functionality of a system while non-functional describe attributes like reliability, maintainability and security, etc.

The system's functional requirements are as follows.

- The mobile records the breathing pattern and movements, using the built-in microphone and accelerometer and stores the results on the SD card of the phone.

- The mobile records the location and transmits the coordinates to the server.

- The password is sent encrypted to the server.

- The system checks for successful logins, failed logins, and successful registrations against the stored entries in the database.

- The MATLAB code accurately processes the audio and movement data.

- The results are stored on the database and displayed on the web portal.

- The GSM successfully sends an SMS alert to the users.

Non-functional requirements for the system dictate that the system is reliable, portable, accurate, maintainable, secure, accessible remotely and easy to use. Non-functional requirements in this system include functionality and mobility.

\section{A. Functionality}

The system is not time-critical, therefore, it does not require the results to be provided to the user in real-time. Hence, data is processed on the server side rather than on the phone. Furthermore, shifting the MATLAB processing to the mobile side will no doubt consume battery energy, and require more time, given the slower 
processor on the phone. In addition, the reliability and maintenance of the system becomes less of a hassle if the processing is done on the server side as algorithms can be easily updated and thresholds can be reconfigured on the remote server (rather than on each user's phone) if the need arises.

\section{B. Mobility}

The mobile application is downloaded onto the patient's phone which allows the patient to travel anywhere as long as he/she is within Wi-Fi or $3 \mathrm{G}$ coverage. The remote live server allows doctors around the world to $\log$ in to the website and check records at any time if they have an internet connection. The continuous communication between patients and physicians is provided by SMS messaging and the internet.

\section{System Testing And Implementation Results}

In order to complete the design and implementation of the proposed Sleep Apnea Monitor application (SAM), the system was successfully tested by following the performance and design requirements as mentioned in Section IV.

The user starts by placing the smart phone on his/her arm, abdomen or near him on the bed (in the latter case, the sensitivity of the in-built accelerometer can be increased to detect small movements or a wireless Bluetooth accelerometer can be used) and start the application by pressing the "Record" button. Both the movement and breathing pattern will be recorded simultaneously during the night. At the end of the sleep study, the application will be stopped (by pressing the "Stop" button). Before the recorded data can be sent, the user needs to register (if he/she is using the application for the first time) and log-in or simply log-in using a valid username and password; this information is sent to the server for authentication purposes, as well as for processing.

The application was tested and the functionality was verified on 55 samples. All sample entries were stored and updated in the database. The preliminary results performed by the server can be retrieved and viewed on the smart phone. Figures 5 and 6 display samples of the accelerometer and voice results, respectively.

If the user is experiencing severe, mild, or no sleep apnea, then Figure 7(a), 7(b), and 7(c) will be displayed on the screen, respectively.

\section{CONCLUSION}

SAM is a mobile application designed to remotely monitor the sleeping patterns of individuals and assess their likeliness to have symptoms of sleep apnea. Even though mobile applications that monitor sleep apnea exist, these applications only monitor one aspect (motion, snoring pattern or oxygen level) as a stand-alone project However, this work is different and innovative in the sense that it combines the different aspects (motion and voice recorder) into one system in order to provide a preliminary diagnosis of user's sleeping pattern anywhere at any time.

This application is targeted at any individual who suspects a sleeping disorder, regardless of his/her age. By using this application, the user can monitor his/her sleep at a very low-cost from the convenience of his/her home, compared to the expensive polysomnographic tests, which require the user to visit a specialized hospital and spend a night in it wearing uncomfortable monitoring equipment.

The system also has a location tracking feature using GSM cell tower triangulation that allows authorized doctors to view locations of the users as well as the severity of their condition. This feature can be customized and markers can be plotted according to gender, age group, employment as well as other factors for research purposes and increase awareness of this growing problem.
With the development of this application we hope to impact the lives of not only sleep apnoeic patients but also those not suffering from this disorder, around the world.

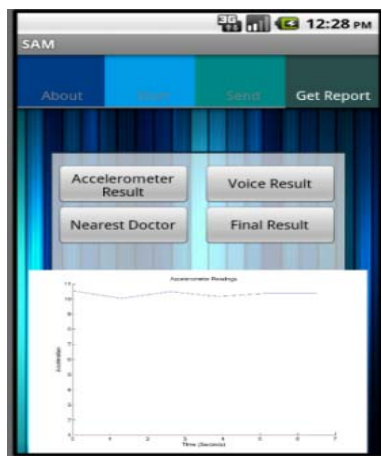

Figure 5. Accelerometer Result.

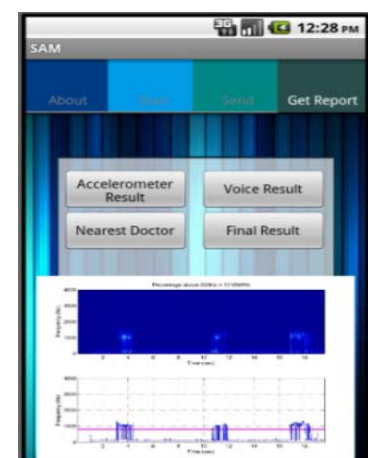

Figure 6. Voice Result.

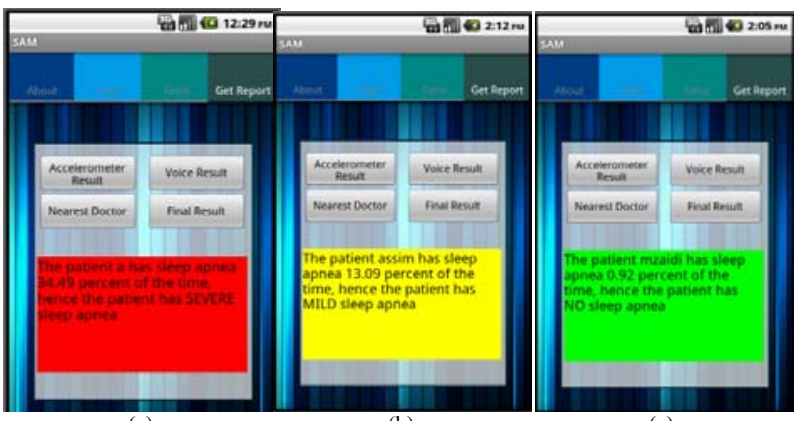

(a)

(b)

(c)

Figure 7. Example showing the results for severe, mild and no sleep apnea.

\section{REFERENCES}

[1] S. Agarwal and C. T. Lau, "Remote Health Monitoring Using Mobile Phones and Web Services," in Telemedicine and e-Health, 16(5), 603607, June 2010

[2] A. Visvanathan, A. Gibb and R. Brady, "Increasing Clinical Presence of Mobile Communication Technology: Avoiding the Pitfalls," in Telemedicine and e-Health, 17(8), 656-661, October 2011.

[3] A. Malhotra, and D. White, "Obstructive Sleep Apnea," in The Lancet, 360(9328), 237-245, July 2002.

[4] "Sleep Apnea," American Sleep Association, September 2007.

[5] "Obstructive Sleep Apnea FAQs," Apnex Medical Inc.

[6] M. Ahmed, N. Patel and I. Rosen, "Portable monitors in the diagnosis of obstructive sleep apnea," in Chest, 132(5), 1672-1677, 2007.

[7] N. Collop et al. "Clinical guidelines for the use of unattended portable monitors in the diagnosis of obstructive sleep apnea in adult patients. Portable Monitoring Task Force of the American Academy of Sleep Medicine," in Journal of Clinical Sleep Medicine, 3(7), 737-747, December 2007.

[8] "Obstructive Sleep Apnea - Diagnosis," University of Maryland Medical Center, 2011.

[9] M. Bsoul, H. Minn and L. Tamil, "Apnea MedAssist: Real-time Sleep Apnea Monitor Using Single-Lead ECG," in IEEE Transactions on Information Technology in Biomedicine, 15(3), 416-427, May 2011.

[10] J. Fiz et al. "Acoustic analysis of snoring sound in patients with simple snoring and obstructive sleep apnea," in The European Respiratory Journal, 9(11), 2365-2370, November 1996.

[11] J. Perez-Padilla et al. "Characteristics of the Snoring Noise in Patients with and without Occlusive Sleep Apnea," in The American Review of Respiratory Disease, 147(3), 635-644, March 1993.

[12] F. Sposaro and G. Tyson, "iFall: An Android Application for Fall Monitoring and Response," in Proc. of the IEEE Int'l Conference of Engineering in Medicine and Biology Society, September 2009. 